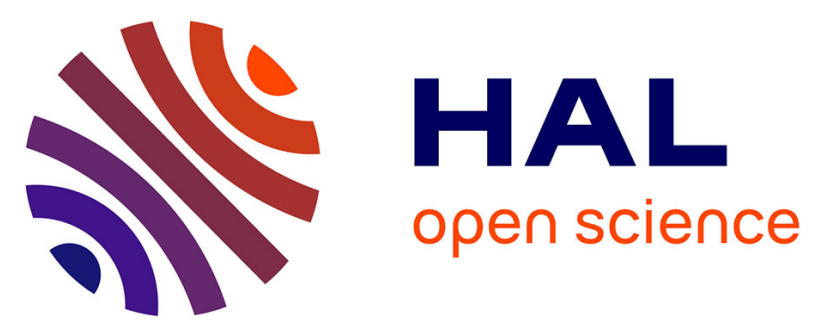

\title{
Body-Surface Atrial Vector Similarity as a New Way to Investigate Atrial Fibrillation Propagation Dynamics
}

Pietro Bonizzi, Stef Zeemering, Joël Karel, Theo Lankveld, Ulrich Schotten, Harry Crijns, Ralf Peeters, Olivier Meste

\section{- To cite this version:}

Pietro Bonizzi, Stef Zeemering, Joël Karel, Theo Lankveld, Ulrich Schotten, et al.. Body-Surface Atrial Vector Similarity as a New Way to Investigate Atrial Fibrillation Propagation Dynamics. Computing in Cardiology, Sep 2021, Brno, Czech Republic. hal-03383348

\section{HAL Id: hal-03383348 \\ https://hal.science/hal-03383348}

Submitted on 18 Oct 2021

HAL is a multi-disciplinary open access archive for the deposit and dissemination of scientific research documents, whether they are published or not. The documents may come from teaching and research institutions in France or abroad, or from public or private research centers.
L'archive ouverte pluridisciplinaire HAL, est destinée au dépôt et à la diffusion de documents scientifiques de niveau recherche, publiés ou non, émanant des établissements d'enseignement et de recherche français ou étrangers, des laboratoires publics ou privés. 


\title{
Body-Surface Atrial Vector Similarity as a New Way to Investigate Atrial Fibrillation Propagation Dynamics
}

\author{
Pietro Bonizzi ${ }^{1}$, Stef Zeemering ${ }^{2,3}$, Joël Karel ${ }^{1}$, Theo Lankveld ${ }^{2,4}$, Ulrich Schotten ${ }^{2,3}$, Harry \\ Crijns $^{3,4}$, Ralf Peeters ${ }^{1}$, Olivier Meste ${ }^{5}$ \\ ${ }^{1}$ Department of Data Science and Knowledge Engineering, Maastricht University, The Netherlands \\ ${ }^{2}$ Department of Physiology, Maastricht University Medical Center, The Netherlands \\ ${ }^{3}$ Cardiovascular Research Institute Maastricht (CARIM), Maastricht University, The Netherlands \\ ${ }^{4}$ Department of Cardiology, Maastricht University Medical Center, The Netherlands \\ ${ }^{5}$ Université Côte d'Azur, CNRS, I3S, France
}

\begin{abstract}
Atrial propagation patterns during atrial fibrillation $(A F)$ can be characterized by a certain degree of recurrence (associated with different types of reentrant circuits that can drive the arrhythmia). In this study, we investigated this recurrent activity at the level of the bodysurface, by measuring the level of similarity between pairs of consecutive atrial vectors. High-density body surface potential maps (120 anterior, 64 posterior electrodes) were recorded in 75 patients in persistent AF. For each patient, atrial vectors were created by taking the samples from all electrodes at each time instant. Similarity between consecutive vectors was measured in terms of the value of the cosine of the angle between two vectors. In all patients, the series of cosine values showed a quasi-periodic behavior, with atrial vectors alternating between phases of slow motion, and phases of fast motion. Moreover, the frequency of this behavior is about twice the AF dominant frequency, which suggests that within one AF cycle there are two phases of slow motion and two of fast motion, alternating. Finally, the amount of slow phases is positively correlated with a higher long-term recurrent behavior of the atrial propagation patterns. This seems to indicate that atrial vectors may provide a new way to noninvasively investigate atrial fibrillation dynamics.
\end{abstract}

\section{Introduction}

Atrial propagation patterns during atrial fibrillation (AF) can be characterize by a certain degree of recurrence [1-6], which can be associated with different types of reentrant circuits that can drive the arrhythmia. The investigation of how well this dynamic behavior of atrial activity (AA) propagation patterns during AF can be noninvasively characterized by analyzing ECG signals is relevant in order to improve AF patient stratification and treatment selection, without resorting to invasive analysis. In this respect, previous studies have shown that propagation of AA during $\mathrm{AF}$ is a process characterized by different short- (or a single cycle) and long-term (related to several cycles of propagation) recurrent behaviours $[8,11]$, while another study suggested that the time-varying spatio-temporal properties of this behavior seem mainly due to a spatially uncoordinated propagation of the AF waveforms [9]. In this study, we investigated this recurrent activity at the level of the body-surface, by measuring the level of similarity between pairs of consecutive body-surface atrial vectors. Results show that body-surface atrial vectors contain information about AA dynamics during AF, and they may represent an alternative way to noninvasively analyze AF dynamics.

\section{Methods}

\subsection{BSPM data and pre-processing}

Body surface potential maps (BSPMs) were recorded in 75 patients in persistent AF, with 120 anterior and 64 posterior leads (ActiveTwo BSM Panels Carbon Electrodes, Biosemi B.V., The Netherlands; Fig. 1). All patients underwent electrical cardioversion, and 32 patients showed $\mathrm{AF}$ recurrence 4 to 6 weeks after procedure. ECGs were sampled at $2048 \mathrm{~Hz}$, and downsampled at $256 \mathrm{~Hz}$. A oneminute segment was selected for each subject, low-quality leads were excluded (low signal-to-noise ratio, poor electrode contact, motion artefacts), and Wilson's Central Terminal was subtracted in line with conventional ECG analysis. After band-pass filtering the signals between 1 and $100 \mathrm{~Hz}$ (3rd order Chebyshev), QRST cancellation was performed using an adaptive singular value decomposition method, inspired by [7], with multiple QRST window templates defined using hierarchical clustering. The extracted 


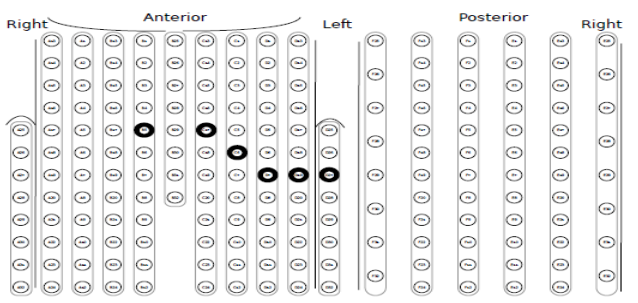

Figure 1. BSPM electrode configuration, comprising 120 anterior leads and 64 posterior leads. Thick circles: default positions of the precordial leads $V_{1}, \ldots, V_{6}$.

AA signals were post-filtered with a zerophase notch filter at $50 \mathrm{~Hz}$ to suppress power line noise, and with a $3 \mathrm{~Hz}$ zerophase highpass filter (3rd order Chebyshev) to remove lowfrequency residuals not related to (persistent) AF.

\subsection{Atrial vectors and similarity signal}

For each patient, atrial vectors were created as follows: assuming that all extracted AA signals from a patient $(\ell$ leads, and $N$ samples) are collected in a matrix $X$ of size $\ell \times N$, each column of $X$ provides an $\ell \times 1$ vector $\mathbf{x}(n)$, $n=1, \ldots, N$, which represents the overall spatial AA from all electrodes at a given time instant. $\mathbf{x}(n)$ can be interpreted as a snapshot of atrial potentials reflected on the body surface at discrete time $n$ (or as a sort of atrial dipole, defined in a space of dimension $\ell$, similar to the 3-D cardiac dipole in vectorcardiography). Similarity between pairs of consecutive vectors (1 sample apart) can then be measured in terms of the value of the cosine of the angle between the two vectors:

$$
\operatorname{sim}_{1}(n)=\frac{\mathbf{x}(n)^{T} \mathbf{x}(n+1)}{\|\mathbf{x}(n)\|_{2}\|\mathbf{x}(n+1)\|_{2}}, n=1, \ldots, N-1 .
$$

$\operatorname{sim}_{1}(n)$ is expected to give values close to 1 (small angles, or high similarity) for spatial AA wave fronts with similar morphologies even when their corresponding amplitudes are different, in order to highlight the presence of self-similar patterns in the data. Fig. 2 provides a schematic representation of the generation of a similarity signal $\operatorname{sim}_{1}(n)$ from body-surface AA signals. As it can be seen in the figure, these signals show a very similar quasi-periodic behavior, with periods of values close to 1 (similar vectors), and very short periods of values closer to 0 (sometimes negative - not in the figure; dissimilar vectors), and quick transitions between the two. This suggests that the vectors alternate between phases of slow motion (values close to 1), and phases of fast motion (values close to 0 ). Therefore, it is interesting to analyze the time and frequency properties of similarity signals, in order to understand if and to what extent they can provide an alterna-

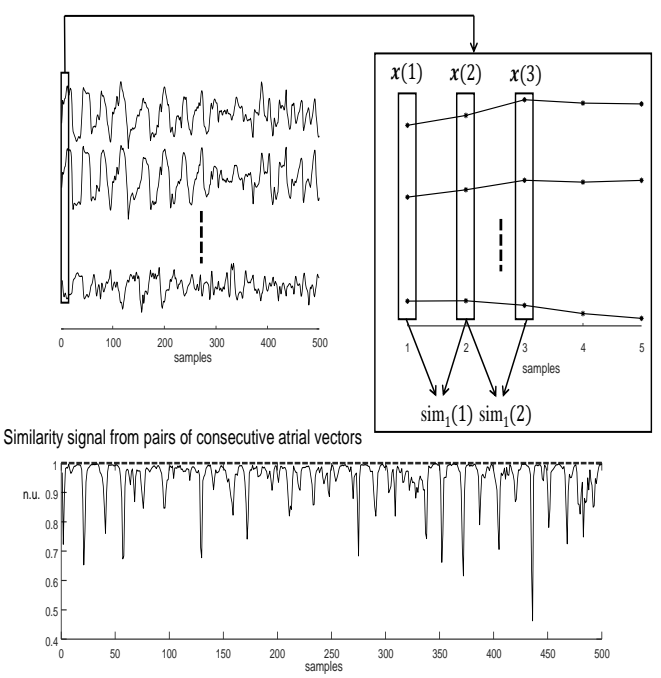

Figure 2. Schematic representation of the construction of a similarity signal $\operatorname{sim}_{1}(n)$ (bottom), starting from AA signals extracted from ECG recordings (top left), and by measuring the level of similarity between consecutive atrial vectors (defined as the samples from all signals at a specific time point; top right).

tive way to look at AA propagation dynamics during atrial fibrillation, as reflected on the body-surface. Moreover, it is also relevant to investigate their relation to the recurrent behavior of those dynamics, which we already described in previous studies [8-11]. Indeed, in [8] we suggested that AA propagation as recorded on the body surface is a process characterized by short- and long-term recurrent behaviors. Namely, an early phase characterized by a decrease of the similarity between an atrial vector and the following so many vectors within approximately one AF cycle, and a later phase characterized by an approximately constant similarity. The long-term recurrent behavior in particular can be considered as a measure of long-term recurrent behaviour of AA propagation in a patient, and it is assumed to be related to the overall AF substrate complexity (with a higher value to be related to a lower degree of electro-structural remodelling) $[9,11]$.

\section{Analyses and Results}

Fig. 3 shows the percent of points close to 1 (phases of slow motion) and close to 0 (phases of fast motion) for all patients. It can be noticed that phases of slow motion are more prevalent in all patients than phases of fast motion (although there is a large variability in the amount of phases of slow motion among patients). We then identified the $3 \%$ of largest absolute vector similarities in each patient, selected the corresponding subset of $k$ columns of matrix $X$, and decomposed the corresponding submatrix 


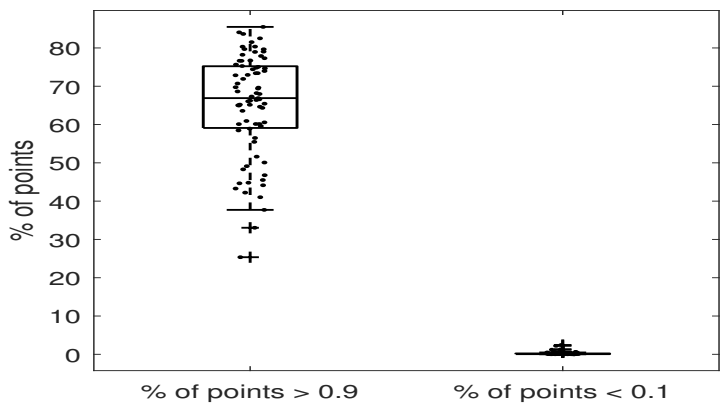

Figure 3. Boxplots of percent of points in a similarity signal close to 1 (threshold 0.9 ) and close to 0 (threshold $0.1)$.

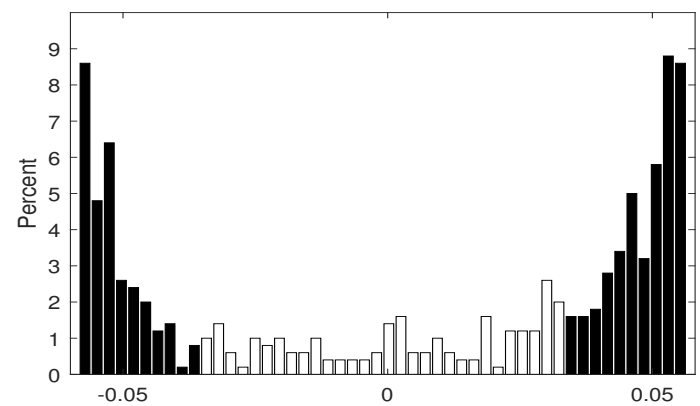

Figure 4. Example of distribution of the values of the first principal component $\mathrm{PC} 1$ of submatrix $\ell \times k$ of $X$ collecting the samples associated with the most relevant vector similarities in a patient from the data set. The distribution is bimodal, with the two main modes located at the extremes (black bars).

of size $\ell \times k$ by means of singular value decomposition. In general, we noticed that the values of the first principal component of the submatrix $\ell \times k$ show a bimodal distribution, with values concentrated at the negative and positive extremes (see Fig. 4, which depicts the distribution of the first principal component for the same patient as in Fig. 2). Spatially, that means that vectors from two consecutive slow regions are in opposition of phase. Fig. 5 shows the percent of points in the first principal component of the submatrix $\ell \times k$ which are located at the extremes of the distribution (first and last ten bins), for all patients. It can be noticed that in all patients at least more than $50 \%$ of the points are located at the extremes of the distribution. When looking at the frequency content of a similarity signal, we noticed that in general such signals show a dominant frequency which is about twice the dominant frequency of the AF in a patient. A representative example is given in Fig. 6, which shows the average power spectral densities of the body-surface AA signals and of the

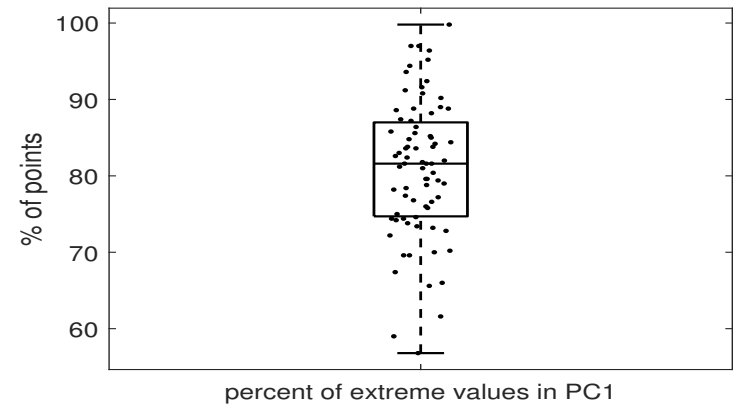

Figure 5. Boxplots of percent of points in the first principal component $\mathrm{PC} 1$ of the submatrix $\ell \times k$ which are located at the extremes of the distribution (first and last ten bins), for all patients.

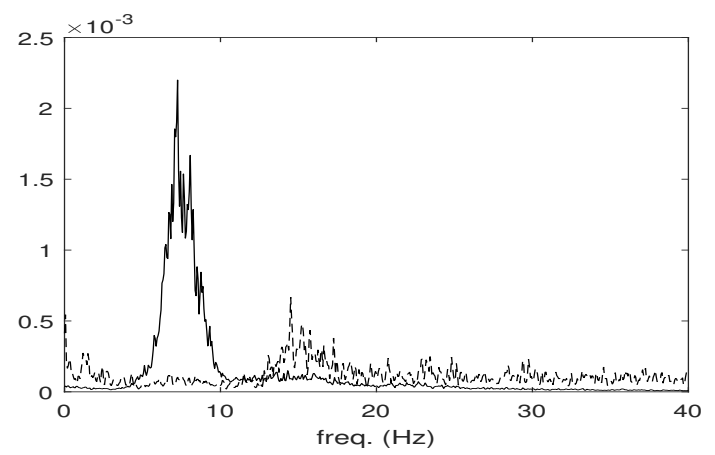

Figure 6. Power spectral densities of the body-surface AA signals (continuous line; average for all signals) and of the similarity signal (dashed line) for the same patient as in Fig. 2.

similarity signal for the same patient as in Fig. 2. Fig. 7 shows the boxplots of dominant frequencies of the AA signals and similarity signals for all patients, which confirms that in general the frequency of the periodic alternation between phases of slow motion and phases of fast motion in a similarity signal is about twice the $\mathrm{AF}$ dominant frequency (13.6(1.8) $\mathrm{Hz}$ vs. 6.6(1.1) Hz, respectively; median(IQR)). Together with the results presented in Fig. 4 and 5, this suggests that within one AF cycle there are two phases of slow motion and two of fast motion, alternating. Finally, we found a positive correlation between the long-term recurrence of the AA during AF, as defined in $[8,9,11]$, and the amount of slow phases in a patient $\left(0.59, p<10^{4}\right)$; Fig. 8; no significant association with electrical cardioversion outcome).

\section{Conclusions}

This study investigated whether AA dynamics during AF can be characterized by measuring the level of similar- 


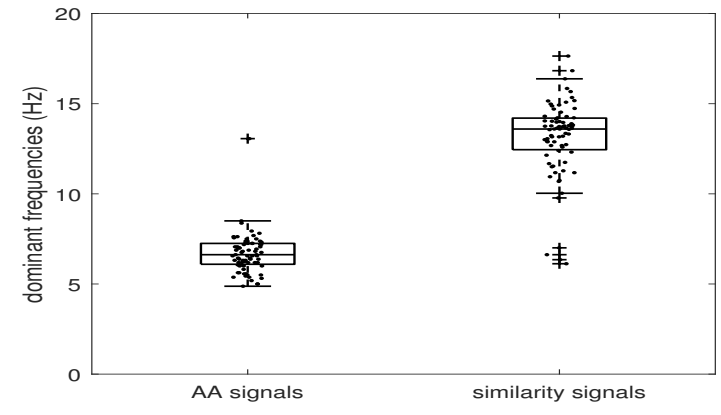

Figure 7. Boxplots of dominant frequencies of the AA signals and similarity signals for all patients.

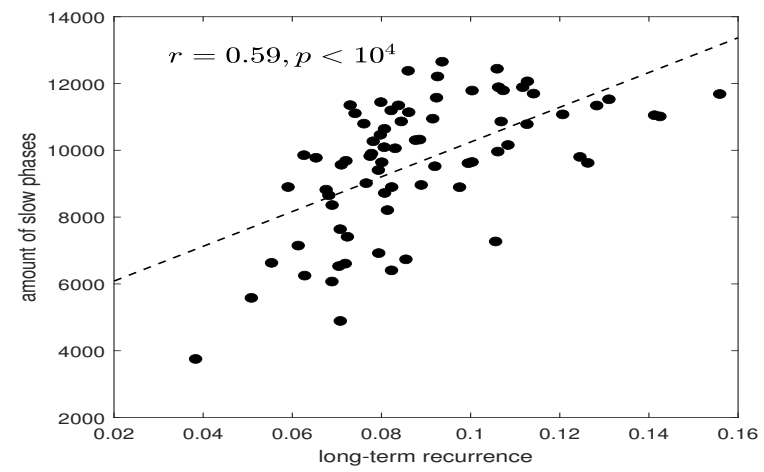

Figure 8. Scatter plot of the amount of slow phases vs. the long-term recurrence. The corresponding line of best fit and the Pearson correlation coefficients $r$ are also shown.

ity between pairs of atrial vectors. The similarity signals obtained by measuring similarity of consecutive atrial vectors showed a quasi-period behavior in all patients, with periods of slow motion (similar vectors) alternating with periods of fast motion (dissimilar vectors), with a frequency of about twice the AF dominant frequency. This suggests that within one AF cycle there are two phases of slow motion and two of fast motion, alternating, with the two phases of slow motion in opposition of phase. In general, we found that there are more phases of slow motion than of fast motion in a patient, and that the amount of time spent in phases of slow motion positively correlates with the long-term recurrent behavior of the atrial propagation patterns. Overall, this seems to suggest that atrial vectors are able to capture to some extent information about AA dynamics during $\mathrm{AF}$, as reflected on the body-surface, and therefore they may represent an alternative way to noninvasively analyze AF dynamics, and the organization of the underlying AA propagation patterns.

\section{References}

[1] Almeida T, Schlindwein F, Salinet J.and Li X, Chu G, Tuan J, Stafford P, Ng G, Soriano D. Characterization of human persistent atrial fibrillation electrograms using recurrence quantification analysis. Chaos An Interdisciplinary Journal of Nonlinear Science 2018;28(8):085710.

[2] van Hunnik A, Zeemering S, Podziemski P, Simons J, Gatta G, Hannink L, Maesen B, Kuiper M, Verheule S, Schotten U. Stationary atrial fibrillation properties in the goat do not entail stable or recurrent conduction patterns. Frontiers in Physiology 2018;9:947.

[3] Ng J, Gordon D, Passman R, Knight B, Arora R, Goldberger J. Electrogram morphology recurrence patterns during atrial fibrillation. Heart Rhythm 2014;11(11):20272034.

[4] Zeemering S, Bonizzi P, Maesen B, Peeters R, U. S. Recurrence quantification analysis applied to spatiotemporal pattern analysis in high-density mapping of human atrial fibrillation. In Conf Proc IEEE Eng Med Biol Soc. 2015; 7704-7.

[5] Zeemering S, van Hunnik A, van Rosmalen F, Bonizzi P, Scaf B, Delhaas T, Verheule S, Schotten U. A novel tool for the identification and characterization of repetitive patterns in high-density contact mapping of atrial fibrillation. Frontiers in Physiology 2020;11:1304.

[6] van Rosmalen F, Maesen B, van Hunnik A, Hermans B, Bonizzi P, Bidar E, Nijs J, Maessen J, Verheule S, Delhaas T, U. U, Zeemering S. Incidence, prevalence, and trajectories of repetitive conduction patterns in human atrial fibrillation. EP Europace 03 2021;23(Supplement 1):i123-i132.

[7] Alcaraz R, Rieta JJ. Adaptive singular value cancellation of ventricular activity in single-lead atrial fibrillation electrocardiograms. Physiol Meas 2008;29:1351-1369.

[8] Meste O, Zeemering S, Karel J, Azman M, Lankveld T, Schotten U, Crijns H, Peeters R, Bonizzi P. Noninvasive Recurrence Quantification Analysis Predicts Atrial Fibrillation Recurrence in Persistent Patients Undergoing Electrical Cardioversion. In Proc. Computing in Cardiology 2016; 43.

[9] Bonizzi P, Zeemering S, Karel J, Azman M, Lankveld T, Schotten U, Crijns H, Peeters R, Meste O. Noninvasive Characterisation of Short- and Long-Term Recurrence of Atrial Signals During Persistent Atrial Fibrillation. In Proc. Computing in Cardiology 2017; 44.

[10] Meste O, Zeemering S, Karel J, Azman M, Lankveld T, Schotten U, Crijns H, Peeters R, Bonizzi P. Use of Normalized Correlation Function to Discriminate Outcome of Persistent Patients Undergoing Electrical Cardioversion. In Proc. Computing in Cardiology 2020; 47.

[11] Bonizzi P, Meste O, Zeemering S, Karel J, Lankveld T, Schotten U, Crijns H, Peeters R. A novel framework for noninvasive analysis of short-term atrial activity dynamics during persistent atrial fibrillation. Medical Biological Engineering Computing 2020;58(9):1933-1945.

Address for correspondence:

Pietro Bonizzi

P.O. Box 616, 6200 MD, Maastricht, The Netherlands

pietro.bonizzi@maastrichtuniversity.nl 